\title{
Article \\ Computational Geometry of Period-3 Hyperbolic Components in the Mandelbrot Set
}

\author{
Young-Hee Geum and Young-Ik Kim *
}

Department of Mathematics, Dankook University, Cheonan 330-714, Korea; conpana@empal.com

* Correspondence: yikbell@dankook.ac.kr

\begin{abstract}
A parametric theoretical boundary equation of a period-3 hyperbolic component in the Mandelbrot set is established from a perspective of Euclidean plane geometry. We not only calculate the interior area, perimeter and curvature of the boundary line but also derive some relevant geometrical properties. The budding point of the period- $3 k$ component, which is born on the boundary of the period- 3 component, and its relevant period- $3 k$ points are theoretically obtained by means of Cardano's formula for the cubic equation. In addition, computational results are presented in tables and figures to support the theoretical background of this paper.
\end{abstract}

Keywords: parameter space; Mandelbrot set; budding point; hyperbolic component; critical orbit

MSC: 65H05; 65H99; 41A25; 65B99

\section{Introduction}

A complex discrete dynamical system with a control parameter $\lambda \in \mathbb{C}$ can be represented by a sequence:

$$
x_{n+1}=\Phi_{\lambda}\left(x_{n}\right), n=0,1,2, \cdots,
$$

where the map $\Phi_{\lambda}$ is a sequence-generating function. We are often interested in the limit behavior or steady-state long-term behavior of such a discrete dynamical system. The following definition presented in [1-3] is reintroduced in consideration of general readers in various fields with elementary knowledge of a dynamical system.

Definition 1. Let $z_{0}$ and $z_{c}$ be in the domain of the sequence-generating function $\phi$. A critical point is a root $z_{c}$ satisfying $0=\left.\frac{d}{d z} \phi(z)\right|_{z=z_{c}}$. The orbit of $z_{0}$ is defined to be the sequence $\left\{\phi^{k}\left(z_{0}\right)\right\}_{k=0}^{\infty}$ with $\phi^{k}$ as the $k$-fold composite map of $\phi$. Then we say the following:

Received: 28 August 2021

Accepted: 27 September 2021

Published: 7 October 2021

Publisher's Note: MDPI stays neutral with regard to jurisdictional claims in published maps and institutional affiliations.

(a) The orbit of a critical point $z_{c}$ is called the critical orbit;

(b) $z_{0}$ has a period $k$ (or is a period- $k$ point of $\phi$ ) if $\phi^{k}\left(z_{0}\right)=z_{0}$, with all distinct $z_{0}, \phi\left(z_{0}\right), \phi^{2}\left(z_{0}\right)$, $\cdots, \phi^{k-1}\left(z_{0}\right)$. If $k=1$, then $z_{0}$ is called a fixed point;

(c) If $z_{0}$ is a period-k point (or $k$-periodic point), then the orbit of $z_{0}$ is called a $k$-periodic orbit (or $k$-cycle);

(d) Let $z_{0}$ be a k-periodic point and denote the eigenvalue of the map $\phi^{k}$ at $z_{0}$ by $\omega=\left.\frac{d}{d z} \phi^{k}(z)\right|_{z=z_{0}}$. Then $z_{0}$ is said to be attractive (attracting), indifferent and repelling, respectively, when $0<|\omega|<1,|\omega|=1$ and $|\omega|>1$;

Copyright: () 2021 by the authors. Licensee MDPI, Basel, Switzerland. This article is an open access article distributed under the terms and conditions of the Creative Commons Attribution (CC BY) license (https:// creativecommons.org/licenses/by/ $4.0 /)$.

(e) If $z_{0}$ is a point some iterate of which is periodic, i.e., if there exists an integer $1 \leq \ell \leq k-2$ satisfying $\phi^{k}\left(z_{0}\right)=\phi^{k-\ell}\left(z_{0}\right)$, then $z_{0}$ is called an eventually periodic (or a pre-periodic) point;

(f) If the orbit of $z_{0}$ contains a subsequence converging to a stable periodic point, then $z_{0}$ is called an asymptotically periodic point;

(g) If $z_{0}$ is not of types $(b),(e),(f)$, then $z_{0}$ is called an aperiodic (or a non-periodic) point. The orbit of a non-periodic point is said to be non-periodic, stochastic or chaotic. 
Such maps $\Phi_{\lambda}$ can be fixed point operators [4] or iterative numerical methods [5-9] to be used in finding the roots for the governing equation $G_{\lambda}(z)=0$ with a control parameter $\lambda$ encountered in many fields of applied sciences and engineering. The well-known Mandelbrot set denoted by $\mathscr{M}$ is indeed a parameter $\lambda$ space that is related to the limit behavior of the discrete dynamical system under the map $\Phi_{\lambda}(z)=z^{2}+\lambda$. It is defined to be a parameter space of $\lambda$ such that the long-term limit behavior of the critical orbit becomes bounded as follows:

$$
\mathscr{M}=\left\{\lambda \in \mathbb{C}: \lim _{\ell \rightarrow \infty} \Phi_{\lambda}^{\ell}(0) \neq \infty\right\}
$$

The geometric shapes of the fixed and period-2 component in $\mathscr{M}$ are well known as a cardioid and a circle, respectively. As a motive to investigate the geometry of a higherperiodic component, we aim to pursue some geometric properties of period-3 components in $\mathscr{M}$ from the viewpoint of Euclidean plane geometry together with the aid of supporting numerical results. We explore a parametric representation of the boundary equations of the period-3 components in $\mathscr{M}$ and pursue bifurcation points as well as other relevant geometric properties of period- $3 k$ hyperbolic components born along the boundaries. A different parametrization of the boundary equations was introduced from a viewpoint of Galois theory in [10].

Further analyses will be treated in three additional sections that follow. Described in Section 2 are preliminary studies on geometric properties of Mandelbrot set $\mathscr{M}$, being focused on the structure of the boundary equations and the relevant budding points of new periodic components arising on the boundary of the fixed (mother-ship or base-ship) component. Section 3 discusses how to establish an exact parametric boundary equation of the period-3 hyperbolic [11] (attracting) components. Finally in Section 4, we summarize with an overall conclusion and state extending the current work in the future.

\section{Preliminary Studies}

Let us consider a limit behavior of a critical orbit under the map $\Phi_{\lambda}(z)=z^{2}+\lambda$. Then the critical point $z_{c}=0$ is uniquely found. Thus the critical orbit is a sequence given by:

$$
0, \Phi_{\lambda}(0), \Phi_{\lambda}^{2}(0), \cdots, \Phi_{\lambda}^{\ell}(0), \cdots,
$$

Namely, it is an infinite sequence that follows:

$$
0, \lambda, \lambda^{2}+\lambda,\left(\lambda^{2}+\lambda\right)^{2}+\lambda,\left(\left(\lambda^{2}+\lambda\right)^{2}+\lambda\right)^{2}+\lambda, \cdots
$$

Consequently, its limit behavior in the complex plane can be characterized as two cases one of which is unbounded and the other bounded. The unbounded case implies $\lim _{\ell \rightarrow \infty}\left|\Phi_{\lambda}^{\ell}(0)\right|=\infty$ for $\lambda \in \mathbb{C}$, whose result will be paid little attention, in view of the fact that $\Phi_{\lambda}(\infty)=\infty$. On the other hand, the bounded case implying $\lim _{\ell \rightarrow \infty}\left|\Phi_{\lambda}^{\ell}(0)\right|<\infty$ will provide a diversity of patterns on its limit behavior, by virtue of the Bolzano-Weierstrass [12] theorem, which ensures the existence of a subsequence of the relevant orbit converging to a finite number $\eta \in \mathbb{C}$ under the map $\Phi_{\lambda}$. This case directly gives rise to the definition of the Mandelbrot set $\mathscr{M}$ indicated by Equation (2).

Indeed, we express $\ell=\mu j+q$ for any $\ell$ with $\mu<\ell, q \in\{0,1,2, \cdots, \mu-1\}$ and $\ell, \mu, j, q \in \mathbb{N}$. The existence of the convergent subsequences allows us to observe a $q$-periodic point $\eta$ satisfying:

$$
\eta=\lim _{\ell \rightarrow \infty} \Phi_{\lambda}^{\ell}(0)=\lim _{j \rightarrow \infty} \Phi_{\lambda}^{\mu j+q}(0)=\Phi_{\lambda}^{q} \circ \lim _{j \rightarrow \infty} \Phi_{\lambda}^{\mu j}(0)=\Phi_{\lambda}^{q}(\eta)
$$

where $\Phi_{\lambda}^{\mu j}(0)=\Phi_{\lambda}^{j} \circ \Phi_{\lambda}^{j} \circ \cdots \circ \Phi_{\lambda}^{j}(0)$ is the $\mu$-fold composite map of $\Phi_{\lambda}^{j}(z)$ evaluated at $z=0$. As $\lambda \in \mathbb{C}$ varies, the critical orbit under $\Phi_{\lambda}$ will approach a $q$-periodic point $\eta=\Phi_{\lambda}^{q}(\eta)$ with some $q \in \mathbb{N}$ based on the discussions we have made thus far. Since 
$\Phi_{\lambda}^{q}-\eta=0$ defines a polynomial in $\lambda$ for any given $q \in \mathbb{N}$, we have no doubt to find such $\lambda$-values contained in a $q$-periodic hyperbolic component, according to the fundamental theorem of algebra. The following lemma will be useful for the proof of Theorem 1 (part (a)) and its proof is omitted owing to the elementary nature.

Lemma 1. Let $t \in \mathbb{R}$. If $t>2$, then $t^{2}-t>t>2$ holds.

Part (a) of the following theorem uses a different form of $\mathscr{M}$ defined by Equation (2) to take advantage of the computational merit.

Theorem 1. The Mandelbrot set $\mathscr{M}=\left\{\lambda \in \mathbb{C}: \lim _{\ell \rightarrow \infty} \Phi_{\lambda}^{\ell}(0) \neq \infty\right\}$ satisfies the following:

(a) $\mathscr{M}=\left\{\lambda \in \mathbb{C}: \Phi_{\lambda}^{k}(0) \leq 2\right.$ for all $\left.k \in \mathbb{N}\right\} \subset\{\lambda \in \mathbb{C}:|\lambda| \leq 2\}$;

(b) $\mathscr{M}$ is compact and connected.

Proof. (a) Suppose that $\lambda \in \mathscr{M}$ and $|\lambda|>2$. Then we find: $\left|\Phi_{\lambda}(0)\right|=|\lambda|>2$. $\left|\Phi_{\lambda}^{2}(0)\right|=$ $\left|\Phi_{\lambda}(0)^{2}+\lambda\right|=\left|\lambda^{2}+\lambda\right| \geq|\lambda|^{2}-|\lambda|>|\lambda|>2$ by identifying $t=|\lambda|$ in Lemma 1 . We find, by induction on $k \geq 3,\left|\Phi_{\lambda}^{k}(0)\right|=\left|\Phi_{\lambda}^{k-1}(0)^{2}+\lambda\right|>|\lambda|^{2}-|\lambda|>|\lambda|>2$. Hence $\left|\Phi_{\lambda}^{k}(0)\right|>|\lambda|>2$ for all $k \in \mathbb{N}$. Now we fix a $k \in \mathbb{N}$. Thus $\left|\Phi_{\lambda}^{k+1}(0)\right|-|\lambda|=\left|\Phi_{\lambda}^{k}(0)^{2}+\lambda\right|-$ $|\lambda| \geq\left|\Phi_{\lambda}^{k}(0)^{2}\right|-2|\lambda|=\left|\Phi_{\lambda}^{k}(0)\right| \cdot\left|\Phi_{\lambda}^{k}(0)\right|-2|\lambda|>2\left(\left|\Phi_{\lambda}^{k}(0)\right|-|\lambda|\right)$. Hence, $\left|\Phi_{\lambda}^{k+m}(0)\right|-$ $|\lambda| \geq 2^{m}\left(\left|\Phi_{\lambda}^{k}(0)\right|-|\lambda|\right)$ for $m \in \mathbb{N}$, which yields $\lim _{m \rightarrow \infty}\left|\Phi_{\lambda}^{k+m}(0)\right|-|\lambda|=\infty$ and thus $\lim _{m \rightarrow \infty}\left|\Phi_{\lambda}^{k+m}(0)\right|=\infty$; such $\lambda \notin \mathscr{M}$ contradictory to the hypothesis. Therefore, $|\lambda| \leq 2$ must hold for $\lambda \in \mathscr{M}$. Now suppose $\lambda \in \mathscr{M}$ and $\left|\Phi_{\lambda}^{k}(0)\right|>2$ for some $k \in \mathbb{N}$. Then similar treatment shown above yields that $\lim _{m \rightarrow \infty}\left|\Phi_{\lambda}^{k+m}(0)\right|=\infty$ implying $\lambda \notin \mathscr{M}$ contradictory to the hypothesis. Hence $\mathscr{M}=\left\{\lambda \in \mathbb{C}: \Phi_{\lambda}^{k}(0) \leq 2\right.$ for all $\left.k \in \mathbb{N}\right\}$. (b) Let $B_{k}=\{\lambda \in \mathbb{C}$ : $\left.\Phi_{\lambda}^{k}(0) \leq 2\right\}$ for a fixed $k \in \mathbb{N}$. Then $B_{k}$ is compact. Since $\Phi_{\lambda}^{k}$ is continuous, $B_{k}$ is closed. $B_{k} \subset\{\lambda \in \mathbb{C}:|\lambda| \leq 2\}$ is bounded. Thus $\mathscr{M}=\cap_{k} B_{k}$ is also compact. Let $\overline{\mathbb{C}}=\mathbb{C} \cup\{\infty\}$. Suppose that $\overline{\mathbb{C}} \backslash \mathscr{M}$ has a bounded component, say, $\mathscr{E}$. Then, by the maximum principle, $\Phi_{\lambda}^{k}(0)$ assumes its maximum on the boundary of $\mathscr{E}$, which is impossible since $\Phi_{\lambda}^{k}(0) \rightarrow \infty$ as $k \rightarrow \infty$ for $\lambda \in \overline{\mathbb{C}} \backslash \mathscr{M}$. Thus $\overline{\mathbb{C}} \backslash \mathscr{M}$ has no bounded components and it is connected. As a consequence, $\mathscr{M}$ is simply connected, which implies that $\mathscr{M}$ is connected.

Lemma 2. Let $\Omega \subset \mathbb{C}$ and $f: \Omega \rightarrow \Omega$ be analytic. Further, let $f$ have a fixed point $\xi \in \Omega$ with $\left|f^{\prime}(\xi)\right|<1$. Then $f$ has a unique fixed point $\xi$ and the sequence $\left\{z_{n+1}=f\left(z_{n}\right)\right\}_{0}^{\infty}$ converges to $\xi$, provided that any $z_{0} \in \Omega$ is given.

Proof. See Lemma 2.5 of [13].

Let $z^{*}$ be a critical point satisfying $f^{\prime}\left(z^{*}\right)=0$, which implies $z^{*} \in \Omega$. As a result of Lemma 2 , taking $z_{0}=z^{*}$ leads us to the following corollary.

Corollary 1. Let $\xi$ be the fixed point described in Lemma 2. Then every critical orbit (which is an orbit of a critical point) of $f$ tends to $\xi$.

\subsection{Boundary of a Period-k Component}

The subsequent discussions on the components will use the notions for satellite and primitive components described in [3]. The primitive components are indeed standing alone themselves.

Figure 1 displays the typical bifurcation geometries with the forms of inscription, circumscription and emergence between two adjacent components $H$ and $W$ in a $\lambda$-parameter space. Observed in $\mathscr{M}$ are frequently circumscription and emergence types of bifurcations.

The value of $\lambda$ where $\boldsymbol{H}$ germinates from $\boldsymbol{W}$ is called the root (budding) point of $\boldsymbol{H}$ [3]. This root point can be considered to be a bifurcation point when viewed from the $W$ side. 
If $\boldsymbol{W}$ is an unbounded component (such as $\mathbb{C} \backslash \mathscr{M}$ ), then a primitive component $\boldsymbol{H}$ emerges at $\lambda$. In the case of the primitive component, the corresponding bifurcation point frequently takes the form of a cusp point, where each tangent of two branches of $\partial H$ (denoting the boundary of $\boldsymbol{H}$ ) is equal. A cusp-like root point of a small copy of $\mathscr{M}$ may arise at a spoke of an antenna $[3,14]$ of $\mathscr{M}$. To track down such bifurcation points in $\mathscr{M}$, we define a $k$-periodic (hyperbolic) component $\boldsymbol{H}_{k}=\left\{\lambda \in \mathbb{C}: \Phi_{\lambda}(z\right.$ has an attracting $k$-cycle $\}$ as a subset of $\boldsymbol{H}$. We further characterize $\boldsymbol{H}_{k}$, with $\Phi_{\lambda}(z)=z^{2}+\lambda$, by the following expression:

$$
\boldsymbol{H}_{k}=\left\{\lambda \in \mathbb{C}: \text { there exist } \xi \text { such that } \Phi_{\lambda}^{k}(\xi)=\xi \text { and }\left|\frac{d}{d z} \Phi_{\lambda}^{k}(z)\right|_{z=\xi(\lambda)}<1\right\} .
$$

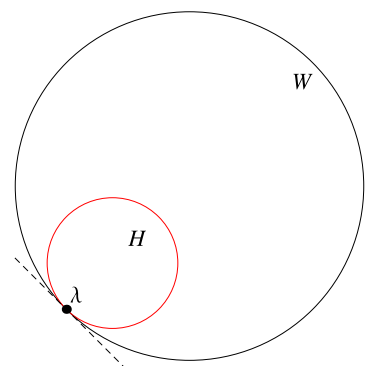

(a) Inscription

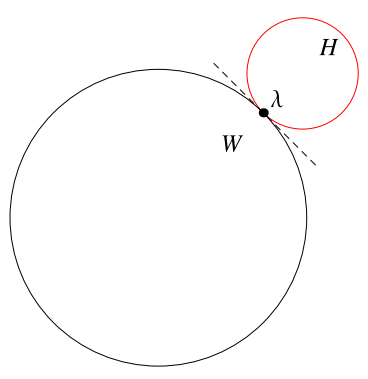

(b) Circumscription

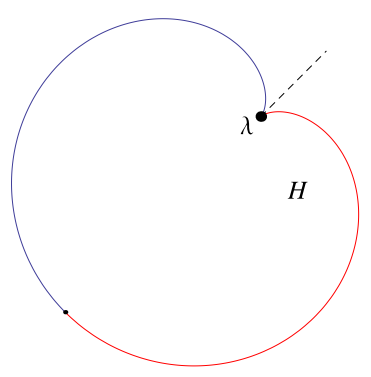

(c) Mergence

Figure 1. Typical bifurcation geometries for budding components.

With the full advantage of Theorem 1 and Lemma 2, the Mandelbrot set $\mathscr{M}$ is constructed and illustrated in Figure 2a with period- $k$ components $\boldsymbol{H}_{k}$ painted by the first 64 colors out of hundreds of colors named in Figure 3. The three period- 3 components respectively denoted by $\boldsymbol{H}_{3}^{\left(s_{1}\right)}$ (satellite), $\boldsymbol{H}_{3}^{(p)}$ (primitive), $\boldsymbol{H}_{3}^{\left(s_{2}\right)}$ (satellite) are displayed counterclockwise as cyan-colored components enclosed in dotted rectangles in Figure $2 \mathrm{a}$.

It is worthwhile to regard the unbounded component as a period- 0 component $\boldsymbol{H}_{0}$. We find that the $k$-periodic point $\xi$ is a root of $2^{k}$-degree polynomial $\Phi_{\lambda}^{k}(z)$ in $z$ with its coefficients depending on $\lambda$ given in $\mathbb{C}$. Clearly, the fundamental theorem of algebra applied to Equation (6) guarantees the existence of such roots $\xi$, the total number of which amounts to be $2^{k}$. The first relation of Equation (6) reveals that $\xi=\xi(\lambda)$ is an analytic function of $\lambda$ [15]. Since $\left.\frac{d}{d z} \Phi_{\lambda}^{k}(z)\right|_{z=\xi(\lambda)}$ is a polynomial in $\xi(\lambda)$, being an analytic function of $\lambda \in \boldsymbol{H}_{k}$, and a continuous function of $\lambda$ in the closure of $\boldsymbol{H}_{k}$, denoted by $\overline{\boldsymbol{H}}_{k}$. By the maximum principle [12], the maximum value of $\left|\frac{d}{d z} \Phi_{\lambda}^{k}(z)\right|_{z=\xi(\lambda)}=1$ occurs on the boundary of $\overline{\boldsymbol{H}}_{k}$. The boundedness of $\boldsymbol{H}_{k}$ for each $k \in \mathbb{N}$ allows the existence of some convergent subsequences of the critical orbit under the map $\Phi_{\lambda}$ with $\lambda \in \boldsymbol{H}_{k}$ in view of the Bolzano-Weierstrass theorem, which will exhibit a periodic, pre-periodic, chaotic or bounded limit behavior. 


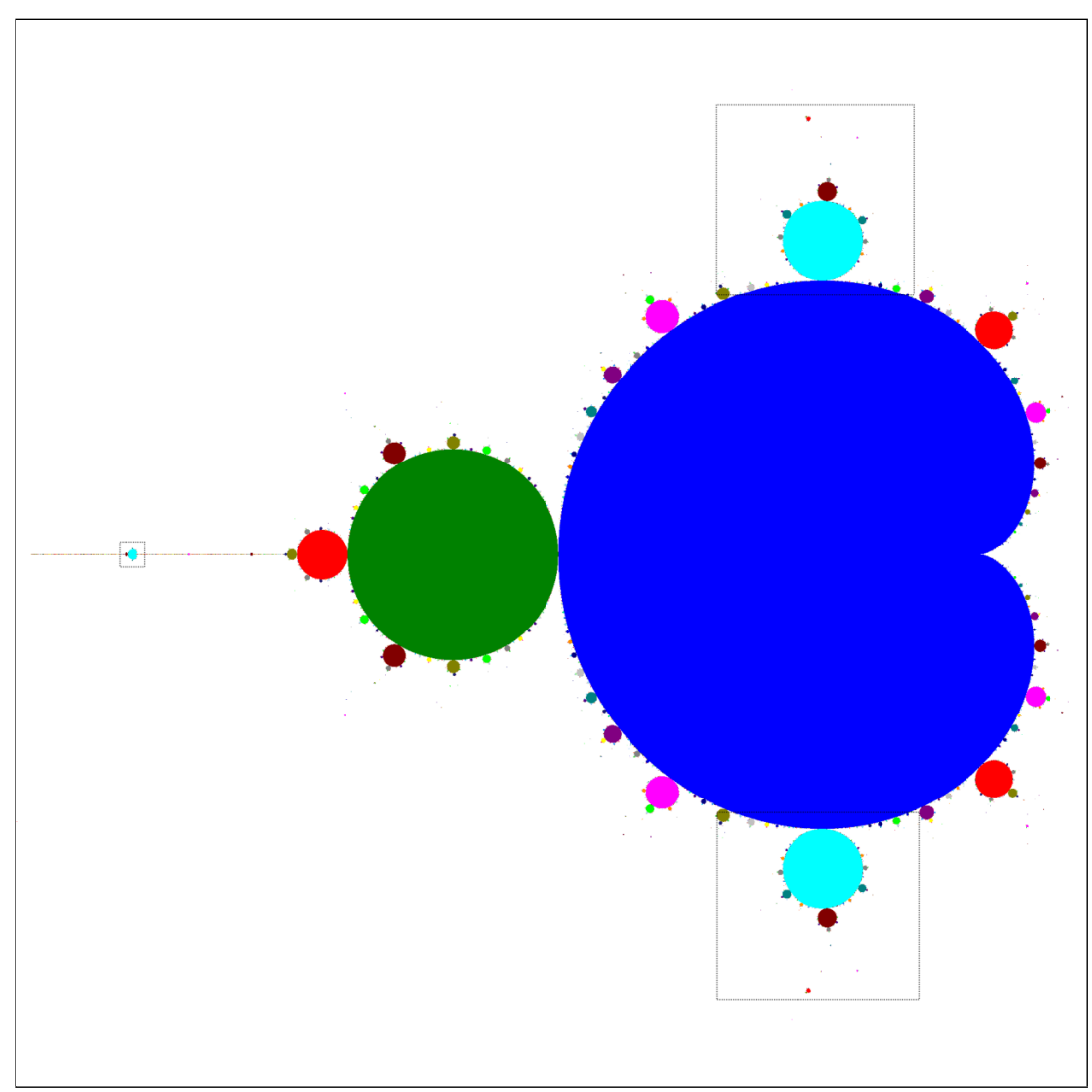

(a) Mandelbrot Set $\mathscr{M}$

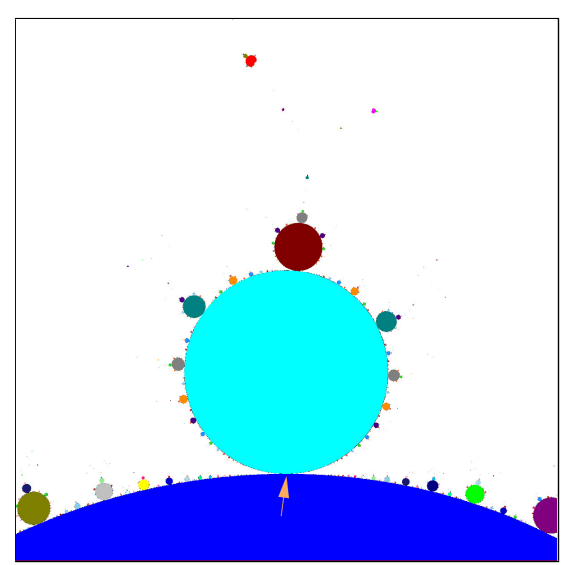

(b) Satellite component $\boldsymbol{H}_{3}^{\left(s_{1}\right)}$

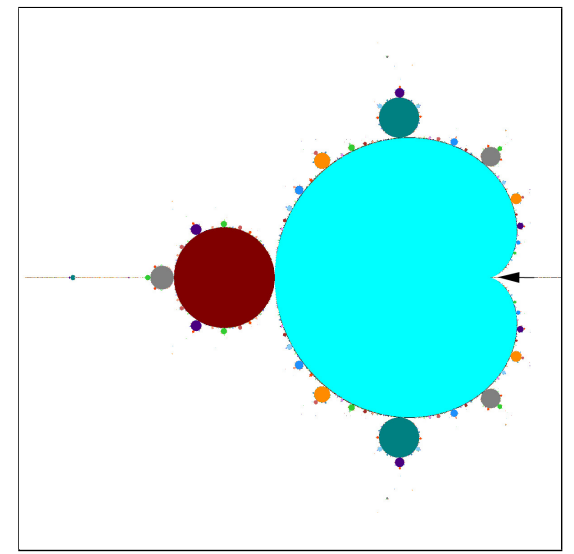

(c) Primitive component $\boldsymbol{H}_{3}^{(p)}$

Figure 2. $\mathscr{M}$, period-3 components $\boldsymbol{H}_{3}^{(s)}$ and $\boldsymbol{H}_{3}^{(p)}$.

Clearly, $\boldsymbol{H}_{1}$ is a main component from which finite-periodic components $\boldsymbol{H}_{k}$ are born. A $\lambda$-dependent critical point $\zeta(\lambda)=0$ found from $\left.\frac{d}{d z} \Phi_{\lambda}^{k}(z)\right|_{z=\zeta(\lambda)}=0$, by identifying $f(z)$ with $\Phi_{\lambda}^{k}(z)$ in Corollary 1, would produce the $\lambda$-parameter space $\mathscr{M}$ whose $\lambda$-value leads us to a long-term dynamical behavior approaching periodic, pre-periodic, non-periodic or chaotic orbits, as $\lambda \in \mathbb{C}$ varies. Our aim is to locate the $\lambda$-values at which $\boldsymbol{H}_{k}$ osculates $\boldsymbol{H}_{1}$. Such $\boldsymbol{H}_{k}$ can be either satellite components born along the boundary $\partial \boldsymbol{H}_{1}$ as can be seen in Figure $1 b$, or primitive components occurring at a spoke of the antenna attached to $\partial \boldsymbol{H}_{1}$. The root points of the primitive components are usually found to be the cusp-like points as can be seen in Figure 1c. 


\begin{tabular}{|c|c|c|c|c|c|c|c|c|}
\hline & $\begin{array}{l}0 \\
\text { Black }\end{array}$ & $\begin{array}{l}1 \\
\text { Blue }\end{array}$ & 2 & $\begin{array}{l}3 \\
\text { Aqua }\end{array}$ & $\begin{array}{l}4 \\
\text { Red }\end{array}$ & $\begin{array}{l}5 \\
\text { Fuchsia }\end{array}$ & $\begin{array}{l}6 \\
\text { Maroon }\end{array}$ & $\begin{array}{l}7 \\
\text { Purple }\end{array}$ \\
\hline & $\begin{array}{l}8 \\
\text { Olive }\end{array}$ & $\begin{array}{l}9 \\
\text { Teal }\end{array}$ & $\begin{array}{l}10 \\
\text { Lime }\end{array}$ & $\begin{array}{l}11 \\
\text { Silver }\end{array}$ & $\begin{array}{l}12 \\
\text { Gray }\end{array}$ & $\begin{array}{l}13 \\
\text { Navy }\end{array}$ & $\begin{array}{l}14 \\
\text { Yellow }\end{array}$ & $\begin{array}{l}15 \\
\text { darkorange }\end{array}$ \\
\hline $\begin{array}{ccccccccc}0 & 1 & 2 & 3 & 4 & 5 & 6 & 7 \\
& 9 & 1 & 11 & 12 & 13 & 1 & 15\end{array}$ & $\begin{array}{l}16 \\
\text { midnight } \\
\text { blue }\end{array}$ & $\begin{array}{l}17 \\
\text { medium } \\
\text { blue }\end{array}$ & $\begin{array}{l}18 \\
\text { indigo }\end{array}$ & $\begin{array}{l}19 \\
\text { skyblue }\end{array}$ & $\begin{array}{l}20 \\
\text { lightblue }\end{array}$ & $\begin{array}{l}21 \\
\text { dodgerblue }\end{array}$ & $\begin{array}{l}22 \\
\text { lightgreen }\end{array}$ & $\begin{array}{l}23 \\
\text { medium } \\
\text { springgreen }\end{array}$ \\
\hline \begin{tabular}{|llllll}
17 & 19 & & 21 & 23 \\
24 & & 26 & 28 & 30
\end{tabular} & $\begin{array}{l}24 \\
\text { lime } \\
\text { green }\end{array}$ & $\begin{array}{l}25 \\
\text { light } \\
\text { cyan }\end{array}$ & $\begin{array}{l}26 \\
\text { deep } \\
\text { skyblue }\end{array}$ & $\begin{array}{l}27 \\
\text { lightskyblue }\end{array}$ & $\begin{array}{l}28 \\
\text { deeppink }\end{array}$ & $\begin{array}{l}29 \\
\text { hotpink }\end{array}$ & $\begin{array}{l}30 \\
\text { indianred }\end{array}$ & $\begin{array}{l}31 \\
\text { orchid }\end{array}$ \\
\hline 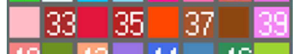 & $\begin{array}{l}32 \\
\text { lightpink }\end{array}$ & $\begin{array}{l}33 \\
\text { brown }\end{array}$ & $\begin{array}{l}34 \\
\text { crimson }\end{array}$ & $\begin{array}{l}35 \\
\text { firebrick }\end{array}$ & $\begin{array}{l}36 \\
\text { orangered }\end{array}$ & $\begin{array}{l}37 \\
\text { sienna }\end{array}$ & $\begin{array}{l}38 \\
\text { lightsteelblue }\end{array}$ & $\begin{array}{l}39 \\
\text { violet }\end{array}$ \\
\hline 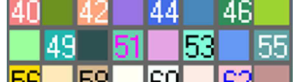 & $\begin{array}{l}40 \\
\text { light } \\
\text { coral }\end{array}$ & $\begin{array}{l}41 \\
\text { olivedrab }\end{array}$ & $\begin{array}{l}42 \\
\text { light } \\
\text { salmon }\end{array}$ & $\begin{array}{l}43 \\
\text { medium } \\
\text { purple }\end{array}$ & royalblue & 45 & seagreen & $\begin{array}{l}47 \\
\text { yellow } \\
\text { green }\end{array}$ \\
\hline 62 & $\begin{array}{l}48 \\
\text { pale } \\
\text { green }\end{array}$ & $\begin{array}{l}49 \\
\text { light } \\
\text { seagreen }\end{array}$ & $\begin{array}{l}50 \\
\text { dark } \\
\text { slategray }\end{array}$ & $\begin{array}{l}51 \\
\text { medium- } \\
\text { aquamarine }\end{array}$ & 52 & 53 & $\begin{array}{l}54 \\
\text { cornflower } \\
\text { blue }\end{array}$ & 55 \\
\hline & $\begin{array}{l}56 \\
\text { gold }\end{array}$ & $\begin{array}{l}57 \\
\text { moccasin }\end{array}$ & $\begin{array}{l}58 \\
\tan \end{array}$ & $\begin{array}{l}59 \\
\text { ivory }\end{array}$ & $\begin{array}{l}60 \\
\text { aliceblue }\end{array}$ & $\begin{array}{l}61 \\
\text { mistyrose }\end{array}$ & $\begin{array}{l}62 \\
\text { pink }\end{array}$ & $\begin{array}{l}63 \\
\text { rosybrown }\end{array}$ \\
\hline
\end{tabular}

Figure 3. Color names for period- $k$ components in $\mathscr{M}$.

\subsection{Budding Points of Period-k Components from a Fixed Component $\boldsymbol{H}_{1}$}

We now focus on the generic case where the iterative map $F$ is analytic. Let $F_{\lambda}(z)$ be analytic in $z \in \mathbb{C}$ with $\lambda \in \mathbb{C}$ as a control parameter; let $z=z(t)$ and $\lambda=\lambda(t)$ be differentiable function of $t$ in an open interval $I=\left(t_{s}, t_{f}\right) \in \mathbb{R}$. With $t$ as a parameter, we consider a parametric curve $\lambda(t)$, which is piecewise differentiable on $I$ and piecewise continuous on the closure $\bar{I}$. Assume that two curves $\lambda=\phi_{1}(t)$ and $\lambda=\phi_{2}(t)$ osculate each other at $t=t^{*}$ with a common tangent line $\tau$ characterized by:

$$
\frac{d \lambda}{d t}=\frac{d \phi_{1}}{d t}=\left.\frac{d \phi_{2}}{d t}\right|_{t=t^{*}}
$$

as shown in Figure 4. For simplicity, we denote $\lambda^{*}=\lambda\left(t^{*}\right)$.

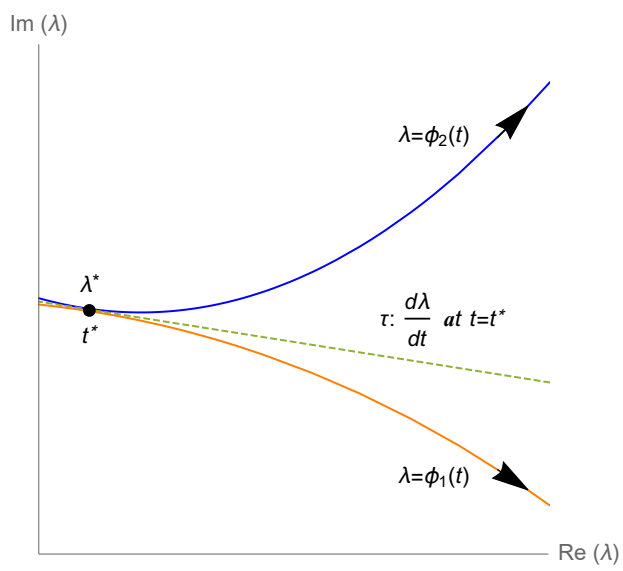

Figure 4. Parametric curves $\lambda(t)$ osculating each other at $t=t^{*}$.

In the space of $\lambda$-control parameter for the map $F_{\lambda}(z)$, let $\lambda(t)=\phi_{1}(t)$ and $\lambda(t)=\phi_{2}(t)$ represent stability boundaries of a given fixed component and of a period- $k$ component for $k \in \mathbb{N}$, respectively. Let $\xi$ be a $k$-periodic point of $F$ for $\lambda=\lambda(t)$. This leads us to the set of equations below:

$$
\left\{\begin{array}{l}
F_{\lambda}(z)=\quad z=\left.F_{\lambda}^{k}(z)\right|_{z=\xi^{\prime}} \\
\frac{\left.d F_{\lambda}^{k}(z)\right)}{d z}=\left.\left(\frac{d F_{\lambda}(z)}{d z}\right)^{k}\right|_{z=\xi}=\beta^{k}, \text { where } \beta=\left.\frac{d F_{\lambda}(z)}{d z}\right|_{z=\zeta} .
\end{array}\right.
$$

Let $\xi=|\xi| e^{i t}$ be a parametric representation for $t \in[0,2 \pi]$. Since we can solve for $\xi=\xi(\lambda)$ from the first row of Equation (7), $\lambda=\lambda(t)$ can be found as a function of the same parameter $t$. It will be shown that relation $\beta^{k}=1$ is satisfied at $\lambda^{*}$ where the two components share the common tangent line as illustrated in Figure 1. 
Suppose that we solve $F_{\lambda}(\xi(t))=\xi(t)$ for $\lambda$ to obtain:

$$
\lambda(t)=\Psi(\xi(t))=\Psi\left(F_{\lambda}^{k}(\xi(t))\right),
$$

with $\Psi$ as a function of $F_{\lambda}$. Clearly, $\lambda(t)$ represents a curve defined by parameter $t$ in $\mathbb{C}$. By means of the derivative chain rule, we can express $\frac{d \lambda}{d t}$ at the fixed point $\xi$ :

$$
\frac{d \lambda}{d t}=\left.\frac{d \Psi(z(t))}{d z} \cdot \frac{d z}{d t}\right|_{z=\xi}=\left.\frac{d \Psi\left(F_{\lambda}^{k}(z(t))\right.}{d z} \cdot \frac{d z}{d t}\right|_{z=\xi}=\left.\frac{d \Psi(z(t))}{d z} \cdot \beta^{k} \cdot \frac{d z}{d t}\right|_{z=\xi^{\prime}}
$$

which implies that $\beta^{k}=1$ in Equation (9). As a result, a period- $k$ component is newly born along the boundary of the fixed component with a fold bifurcation [16].

Indeed, Equation (8) can be solved for the case that $F_{\lambda}(\xi(t))$ is a bivariate rational function of both variables $\xi$ and $\lambda$ since $\Psi$ can be found as a rational function by the fundamental theorem of algebra. Clearly, $\frac{d \lambda}{d t}$ is shared by the common tangent line osculating the two adjacent fixed and period- $k$ components at the point $\xi(t)$.

We solve $\beta^{k}=1$ for $\beta$ in terms of $\lambda$ to obtain:

$$
\beta(\lambda)=\left.\frac{d F_{\lambda}(z)}{d z}\right|_{z=\xi(\lambda)}=e^{i 2 \pi \ell / k}, \text { for } \ell \in\{0,1,2, \cdots, k-1\},
$$

where $\operatorname{gcd}(\ell, k)=1$ if $\ell \neq 0$. Given $k$ and $\ell$, we can solve Equation (10) for $\lambda$, which is called the budding point of period- $k$ point from a fixed component. This budding point $\lambda$ is denoted by $\lambda_{\ell, k}$ and may be regarded as the $\ell / k$-bifurcation point or $\ell / k$-root point.

By an inspection of bifurcation characteristics based on the location of $\beta$-values on the unit circle, we find that the values of $(\ell, k)=(0,1)$ and $(\ell, k)=(1,2)$ yield the fold bifurcation and the flip bifurcation, respectively. Besides, all other values of $(\ell, k)$ lead us to the Neimark-Sacker bifurcation.

\section{Constructing Boundary Equations of Period-3 Components $\mathrm{H}_{3}$}

\subsection{Parametric Equations of $\partial \boldsymbol{H}_{3}$ and Bifurcation Points}

In this subsection, we firstly establish the boundary equation of attracting component $H_{3}$ associated with a typical $\lambda$-dependent period-3 point $z$ given by a root of $\Phi_{\lambda}^{3}(z)=z$. Identifying $F_{\lambda}(z)=\Phi_{\lambda}^{3}(z)$ leads us to an application of preceding discussions treated in Section 2.2. During the analysis, we will pursue the existence of three period-3 components $\boldsymbol{H}_{3}$, each boundary of which is made up of two piecewise analytic branches. Extensive investigation of the boundary curve as well as bifurcation points will be secondly discussed in the remaining subsection. Consulting Equation (7), we find that the period-3 point $z$ satisfies a set of equations with $\lambda \in \partial H_{3}$ as follows:

$$
\begin{cases}\Phi_{\lambda}^{3}(z) & =\left(\left(z^{2}+\lambda\right)^{2}+\lambda\right)^{2}+\lambda=z \\ \left|\frac{d}{d w} \Phi_{\lambda}^{3}(w)\right|_{w=z} & =\left|2^{3}\left(\left(z^{2}+\lambda\right)^{2}+\lambda\right)\left(z^{2}+\lambda\right) z\right|=1\end{cases}
$$

We reconstruct the second row of Equation (11) with a real parameter $t \in[0,2 \pi$ ):

$$
\left(\left(z^{2}+\lambda\right)^{2}+\lambda\right)\left(z^{2}+\lambda\right) z=\frac{1}{8} e^{i t}=a,
$$

where $a$ is introduced for short notations in the subsequent analysis.

From the first row of Equation (11), some algebraic manipulations lead us to:

$$
0=\left(z^{2}+\lambda-z\right) \cdot\left\{\left(z^{2}+\lambda+z+1\right)\left[\left(z^{2}+\lambda\right)^{2}+\lambda+z\right]+1\right\} .
$$

Since $\left(z^{2}+\lambda-z\right)=0$ reflects fixed points, period-3 points $z$ should satisfy

$$
\left(z^{2}+\lambda+z+1\right)\left[\left(z^{2}+\lambda\right)^{2}+\lambda+z\right]+1=0 .
$$


Let $K_{6}=\left(z^{2}+\lambda+z+1\right)\left[\left(z^{2}+\lambda\right)^{2}+\lambda+z\right]+1$ denote the left side of Equation (14). Rewriting Equations (12) and (14) yields the two equations below:

$$
\begin{gathered}
1+z+z^{2}+z^{3}+z^{4}+z^{5}+z^{6}+ \\
\left(1+2 z+3 z^{2}+2 z^{3}+3 z^{4}\right) \lambda+\left(2+z+3 z^{2}\right) \lambda^{2}+\lambda^{3}=0 . \\
z^{7}+\left(z^{3}+3 z^{5}\right) \lambda+\left(z+3 z^{3}\right) \lambda^{2}+\lambda^{3}-a=0 .
\end{gathered}
$$

Multiplying Equation (15) by $(1-z)$ and adding Equation (16) together, we obtain:

$$
1-a+\left(1+z+z^{2}+z^{4}\right) \lambda+2\left(1+z^{2}\right) \lambda^{2}+\lambda^{3}=0 .
$$

Lemma 3. Let $\lambda \in \partial H_{3}$. Then the following relation holds:

$$
\lambda^{3}+2 \lambda^{2}+\lambda(1-a)+(1-a)^{2}=0 .
$$

Proof. From Equation (17), it follows that $1-a=-\lambda\left(1+z+z^{2}+z^{4}+2\left(1+z^{2}\right) \lambda+\lambda^{2}\right)$. Hence, $(1-a)^{2}=K_{6} \cdot\left(\lambda^{2}\left(2-z+z^{2}+\lambda\right)+\lambda^{2}\left(-1+z+z^{2}+z^{4}+\lambda+2 z^{2} \lambda+\lambda^{2}\right)=\lambda^{2}(-1+\right.$ $\left.z+z^{2}+z^{4}+\lambda+2 z^{2} \lambda+\lambda^{2}\right)$, using the fact that $K_{6}=0$ from Equation (14). A direct substitution of these results into the left side of Equation (18) favorably yields a value of zero after canceling out terms associated with $z$, which proves the assertion.

Lemma 3 states that three $\lambda$-values exist for three period- 3 components, each of which defines a boundary curve in terms of $a=\frac{1}{8} e^{i t}$ with a parameter $t \in[0,2 \pi)$. Let $\bar{\lambda}$ denote the complex conjugate of $\lambda$. Then due to the fact that $\overline{\lambda(a)}=\lambda(\bar{a})$ holds, $\bar{z}$ (the complex conjugate of $z$ ) is also a period-3 point for $\bar{\lambda}$. This tells us that period-3 components arise symmetrically with respect to the real-axis of $\mathbb{C}$. Consequently, the boundary curves $\lambda$ of period-3 components are given by Equation (18). As $a$ varies along the circle of radius $1 / 8$ centered at $(0,0)$, it traces two half circles, which are analytic branches formed by parameters $t$ ranging from $0 \leq t<\pi$ and $\pi \leq t<2 \pi$, respectively, assuming the negative real axis as a branch cut. Hence each of three $\lambda$-values has two analytic branches which may behave differently for $t \in[0, \pi)$ and $t \in[\pi, 2 \pi)$.

Using Cardano's formula $[17,18]$, we now solve Equation (18) exactly to obtain three solutions $\lambda^{(j)}(t)$ for $j \in\{1,2,3\}$, in terms of parameter $t \in[0,2 \pi)$, with the aid of symbolic computation of Mathematica [19], as follows:

$$
\left\{\begin{array}{l}
\lambda^{(1)}(t)=-\frac{1}{3}\left(2-\frac{2^{1 / 3}(1+3 a)}{\sigma}-2^{-1 / 3} \sigma\right) \\
\lambda^{(2)}(t)=-\frac{1}{3}\left(2+\frac{2^{-2 / 3}(1+i \sqrt{3})(1+3 a)}{\sigma}+2^{-4 / 3}(1-i \sqrt{3}) \sigma\right) \\
\lambda^{(3)}(t)=-\frac{1}{3}\left(2+\frac{2^{-2 / 3}(1-i \sqrt{3})(1+3 a)}{\sigma}+2^{-4 / 3}(1+i \sqrt{3}) \sigma\right)
\end{array}\right.
$$

with $i^{2}=-1, \sigma=\left(-25+36 a-27 a^{2}+3 \sqrt{3}|1-a| \sqrt{23-22 a+27 a^{2}}\right)^{1 / 3}$ and $a=\frac{1}{8} e^{i t}, 0 \leq$ $t \leq 2 \pi$. Observe that $\overline{\lambda^{(2)}(t)}=\lambda^{(3)}(t)$ holds.

We denote the two analytic branches of $\lambda^{(j)}(t)$ by $\lambda_{a}^{(j)}(t)$ and $\lambda_{b}^{(j)}(t)$ for $j \in\{1,2,3\}$ as follows:

$$
\begin{cases}\lambda_{a}^{(j)}(t)=\lambda^{(j)}(t), & \text { for } t \in[0, \pi), \\ \lambda_{b}^{(j)}(t)=\lambda^{(j)}(t), & \text { for } t \in(\pi, 2 \pi] .\end{cases}
$$


It is necessary to check the value of each solution $\lambda^{(j)}(t)$ for $j \in\{1,2,3\}$ near $0, \pi$ and $2 \pi$.

$$
\begin{aligned}
& \lambda^{(1)}(0)=\frac{-1+3 i \sqrt{3}}{8}, \quad \lambda^{(1)}\left(\pi^{-}\right)=-0.115735+0.837999 i, \\
& \lambda^{(1)}(\pi)=-0.115735-0.837999 i, \quad \lambda^{(1)}\left(2 \pi^{-}\right)=\frac{-1-3 i \sqrt{3}}{8}, \\
& \overline{\lambda^{(1)}\left(\pi^{-}\right)}=\lambda^{(1)}(\pi), \quad \overline{\lambda^{(1)}(0)}=\lambda^{(1)}\left(2 \pi^{-}\right), \\
& \lambda^{(2)}(0)=\frac{-1-3 i \sqrt{3}}{8}, \quad \quad \lambda^{(2)}\left(\pi^{-}\right)=-0.115735-0.837999 i, \\
& \lambda^{(2)}(\pi)=-1.76853, \quad \quad \lambda^{(2)}\left(2 \pi^{-}\right)=-1.75, \\
& \lambda^{(3)}(0)=-1.75, \quad \lambda^{(3)}\left(\pi^{-}\right)=-1.76853, \\
& \lambda^{(3)}(\pi)=-0.115735+0.837999 i, \quad \lambda^{(3)}\left(2 \pi^{-}\right)=\frac{-1+3 i \sqrt{3}}{8},
\end{aligned}
$$

where $a^{+}$and $b^{-}$imply the respective limits from the above of $a$ and the below of $b$. As expected, each of solution branches is disconnected at the singular point $t=\pi$.

Further refined computations readily yield:

$$
\left\{\begin{array}{lll}
\overline{\lambda_{a}^{(1)}(t)}=\lambda_{a}^{(2)}(t), & & \text { for } t \in[0, \pi), \\
\overline{\lambda_{b}^{(1)}(t)}=\lambda_{b}^{(3)}(t), & & \text { for } t \in[\pi, 2 \pi), \\
\overline{\lambda_{a}^{(3)}(t)}=\lambda_{b}^{(2)}(t+\pi), & & \text { for } t \in[0, \pi) .
\end{array}\right.
$$

Solution branches are illustrated in Figure 5, where horizontal and vertical axes labels represent $\Re\left(\lambda^{(j)}(t)\right)$ and $\left.\Im\left(\lambda^{(j)}\right)(t)\right)$, respectively. All remaining figures throughout the paper have the same axes labels.

Observing that $\lambda^{(j)}(t),(1 \leq j \leq 3)$ is a piecewise analytic function of $t \in[0,2 \pi)$, along with the symmetry of $\partial \boldsymbol{H}_{3}$, we characterize its three closed boundary curves $\mathcal{B}_{j}=\left\{\gamma_{j}(t)\right.$, $t \in[0,2 \pi)\}$ for $j \in\{1,2,3\}$, as follows:

$$
\gamma_{1}(t)=\left\{\begin{array}{l}
\lambda_{a}^{(1)}(t), t \in[0, \pi), \\
\lambda_{b}^{(3)}(t), t \in[\pi, 2 \pi),
\end{array} \quad \gamma_{2}(t)=\overline{\gamma_{1}(t)}, \gamma_{3}(t)=\left\{\begin{array}{l}
\lambda_{3}(t), t \in[0, \pi), \\
\lambda_{2}(t), t \in[\pi, 2 \pi) .
\end{array}\right.\right.
$$

In general, each $\gamma_{j}(t),(1 \leq j \leq 3)$ is made up of piecewise analytic arcs with singularities at $t=0$ and $t=\pi$. Figure 6 evidently illustrates the three functions $\gamma_{j}(t),(1 \leq j \leq 3)$ for $t \in[0,2 \pi)$. Let $\gamma_{j}(t)$ for $t \in[0,2 \pi)$ trace a closed plane curve denoted by $\mathcal{B}_{j}$ forming $\partial \boldsymbol{H}_{3}$. The symmetry of $\boldsymbol{H}_{3}$ is clearly visible by the symmetry of $\mathscr{M}$ with respect to the horizontal axis. The blue and magenta arcs represent analytic branches for $t \in[0, \pi)$ and for $t \in[\pi, 2 \pi)$, respectively. The dotted rectangles in Figure 2a clearly display the three cyan-colored period-3 components. 


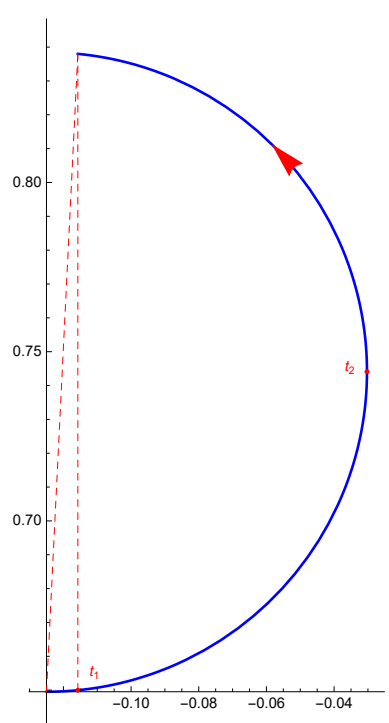

(a) $\lambda_{a}^{(1)}$

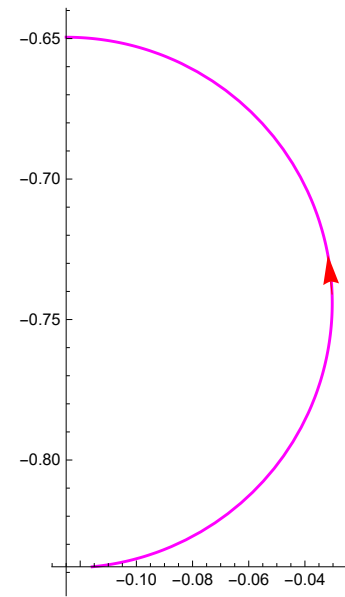

(b) $\lambda_{b}^{(1)}$

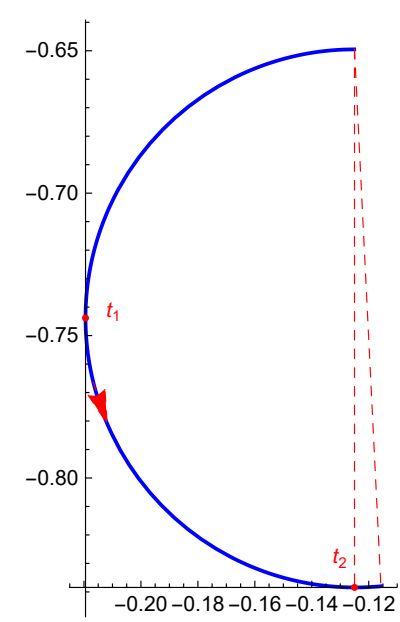

(c) $\lambda_{a}^{(2)}$

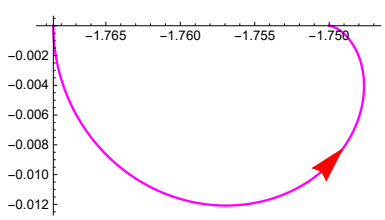

(d) $\lambda_{b}^{(2)}$

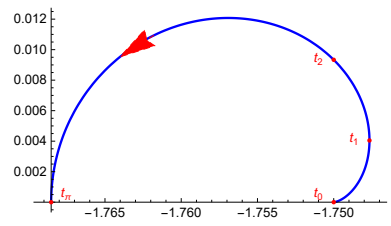

(e) $\lambda_{a}^{(3)}$

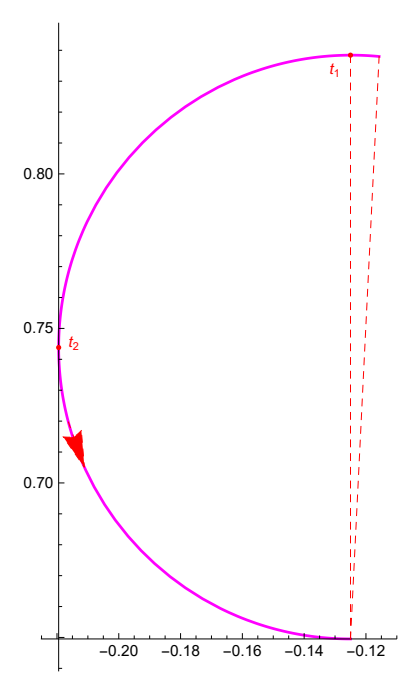

(f) $\lambda_{b}^{(3)}$

Figure 5. Solution Branches of $\lambda^{(j)}(t)$.

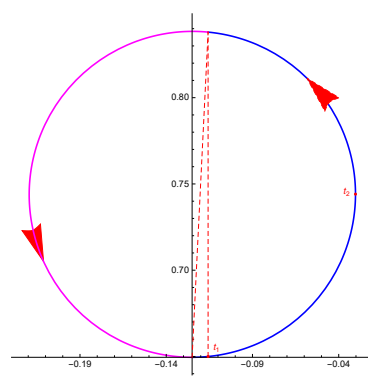

(a) $\mathcal{B}_{1}$

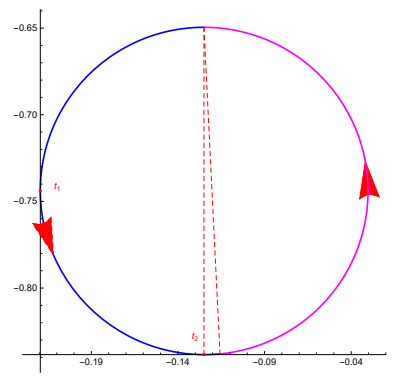

(b) $\mathcal{B}_{2}$

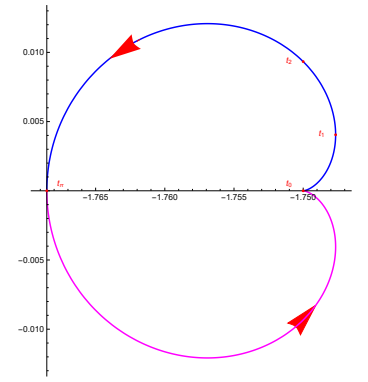

(c) $\mathcal{B}_{3}$

Figure 6. Boundary curves of the three red fixed components $\mathscr{R}^{f}$.

\subsection{Typical Geometric Properties of $\mathcal{B}_{j}$ and Budding Phenomena Occurring on $\partial \mathcal{B}_{j}$}

In this subsection, typical geometric properties including curvatures, perimeters and interior areas of the closed boundary curves $\mathcal{B}_{j}$ will be firstly investigated from a perspective of Euclidean planar curves together with supported numerical results. Budding phenomena related to period-3 points and the relevant bifurcation points on the boundary 
of $\mathcal{B}_{j}$ will be secondly discussed. Let $x_{j}(t)=\Re\left(\gamma_{j}\right)(t)$ and $y_{j}(t)=\Im\left(\gamma_{j}\right)(t)$ denote the real and imaginary part of $\lambda^{(j)}(t)$,respectively. We are more devoted to computing the arc lengths of $\mathcal{B}_{j}$ for $1 \leq j \leq 3$ with approximately 10 digits of accuracy:

$$
\begin{aligned}
& \left|\widehat{\mathcal{B}}_{1}\right|=\int_{0}^{\pi} \sqrt{x_{1}^{\prime}(t)^{2}+y_{1}^{\prime}(t)^{2}} d t+\int_{\pi}^{2 \pi} \sqrt{x_{3}^{\prime}(t)^{2}+y_{3}^{\prime}(t)^{2}} \approx 0.5938968502, \\
& \left|\widehat{\mathcal{B}}_{2}\right|=\left|\widehat{\mathcal{B}}_{1}\right|, \\
& \left|\mathcal{\mathcal { B }}_{3}\right|=\int_{0}^{\pi} \sqrt{x_{3}^{\prime}(t)^{2}+y_{3}^{\prime}(t)^{2}} d t+\int_{\pi}^{2 \pi} \sqrt{x_{2}^{\prime}(t)^{2}+y_{2}^{\prime}(t)^{2}} \approx 0.07433268157,
\end{aligned}
$$

Let $A_{j 1}$ denote the interior area enclosed by an arc $\gamma_{j}(t),(0 \leq t<\pi)$ and a line segment connecting the end points of the arc. Similarly, let $A_{j 2}$ denote the interior area enclosed by an arc $\gamma_{j}(t),(\pi \leq t<2 \pi)$ and a line segment connecting the end points of the arc. Then we can find the interior area $A_{j}=A_{j 1}+A_{j 2}$ for $j \in\{1,2,3\}$ enclosed by $\mathcal{B}_{j}$. We typically find the $A_{11}$ only, since the remaining $A_{j k},(1 \leq j \leq 3,1 \leq k \leq 2)$ can be found in a similar manner.

Let us closely look at Figure 5a. Let $t_{1}$ be the parameter value crossing the arc and the line parallel to $y$-axis passing through the point $\gamma_{1}\left(\pi^{-}\right)$and $t_{2}$ be the parameter value for the maximal $x_{1}(t)$ with $t \in[0, \pi)$. Then

$$
A_{11}=\int_{t_{2}}^{\pi} y_{1}(t) x_{1}^{\prime}(t) d t-\int_{t_{1}}^{t_{2}} y_{1}(t) x_{1}^{\prime}(t) d t+\int_{0}^{t_{1}}\left[y(t) x^{\prime}(t)-y_{1}(t) x_{1}^{\prime}(t)\right] d t \approx 0.01318346626,
$$

where $x(t)+i y(t),\left(0 \leq t \leq t_{1}\right)$ represents a parametric equation of the line segment connecting the two end points $\gamma_{1}(0)$ and $\gamma_{1}\left(\pi^{-}\right)$. Similar computations yield the remaining areas as follows:

$$
A_{12} \approx 0.01488453547, A_{21}=A_{12}, A_{22}=A_{11}, A_{31} \approx 0.0002032073235, A_{32}=A_{31} .
$$

As a result, we obtain the interior areas:

$$
A_{1}=0.02806800174, A_{2}=A_{1}, A_{3}=0.0004064146471 .
$$

An elementary theory of geometry tells us that the Frenet (unsigned) curvature $\kappa$ of a parametric curve $\gamma(t)=x(t)+i y(t)$ is given by

$$
\kappa=\frac{\left|x^{\prime \prime}(t) y^{\prime}(t)-x^{\prime}(t) y^{\prime \prime}(t)\right|}{\left[x^{\prime}(t)^{2}+y^{\prime}(t)^{2}\right]^{3 / 2}} .
$$

The $\kappa$-values of $\mathcal{B}_{j}$ calculated when $t=\frac{\pi}{4}, \frac{\pi}{3}, \frac{\pi}{2}, \frac{2 \pi}{3}, \frac{3 \pi}{4}$, and the average curvatures are shown in Table 1, which additionally lists the bounding areas and perimeters. The following lemma immediately follows from the geometric properties listed in Table 1.

Table 1. Geometric characteristics of Plane Curves $\mathcal{B}_{i}, \mathcal{T}$ and $\mathcal{H}$.

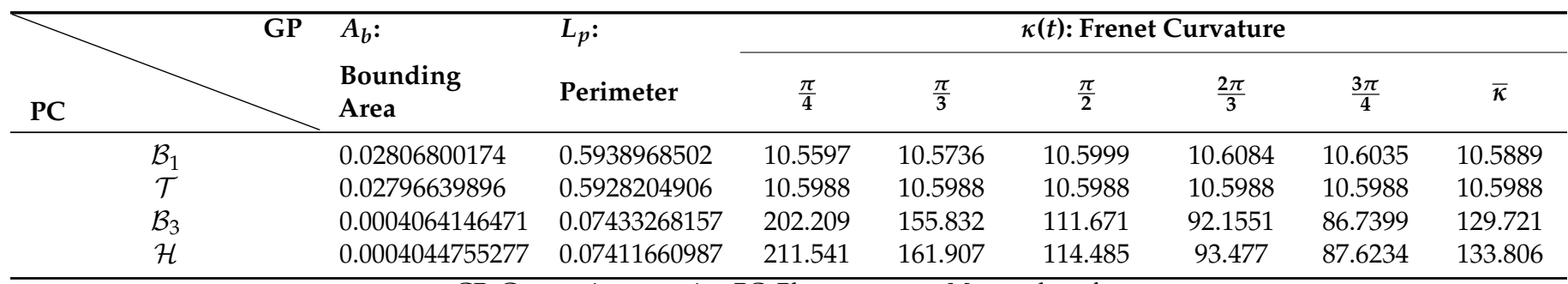

GP: Geometric properties, PC: Plane curves, $\bar{\kappa}$ : Mean value of $\kappa$.

Lemma 4. The shapes of $\mathcal{B}_{i}(1 \leq i \leq 3)$ have the following characteristics:

(i) The boundary of $\mathcal{B}_{1}$ or $\mathcal{B}_{2}$ is not a perfect circle;

(ii) The boundary of $\mathcal{B}_{3}$ is not a perfect cardioid.

Remark 1. Let $B$ be a point $\gamma_{1}\left(2 \pi^{-}\right)$and $A$ be a point on $\mathcal{B}_{1}$ crossing with a vertical line passing through $B$. Consider a circle $\mathcal{T}$ of radius $r_{M}$ given by $\mathcal{T}=\left\{\left(a_{M}+r_{M} \cos t, b_{M}+r_{M} \sin t\right), t \in\right.$ 
$[0,2 \pi)\}$ with a center $\left(a_{M}, b_{M}\right)$, which is the mid point of pints $A$ and $B$. Careful computations yield $a_{M}=-0.125, b_{M}=0.7439727234$ and $r_{M}=0.09435031145$. Let $\rho_{1}(t)=\left|\gamma_{1}(t)-\left(a_{M}+i b_{M}\right)\right|$ be the distance from the center $\left(a_{M}, b_{M}\right)$ to a point $\gamma_{1}(t)$ at a location $t$. The minimum value 0.09435031145 of $\rho_{1}(t)$ occurs at $t=3.937659013$. Consequently, after elaborate computations, we have $\rho_{1}(t) \geq r_{M}$ for all $t \in[0,2 \pi)$. Hence, we find that $\mathcal{B}_{1}$ closely resembles as well as surrounds $\mathcal{T}$. Let us define a cardioid $\mathcal{H}=\{(a+\rho(1-\cos t) \cos t, \rho(1-\cos t) \sin t), t \in[0,2 \pi)\}$, where $a=\gamma_{3}(0)=-1.75, \rho=\left(\gamma_{3}(0)-\gamma_{3}\left(\pi^{-}\right)\right) / 2=0.0185291524$. In view of geometric properties in Table $1, \mathcal{H}$ closely resembles $\mathcal{B}_{3}$ with exactly the same diametral chords (Figure 7 ).

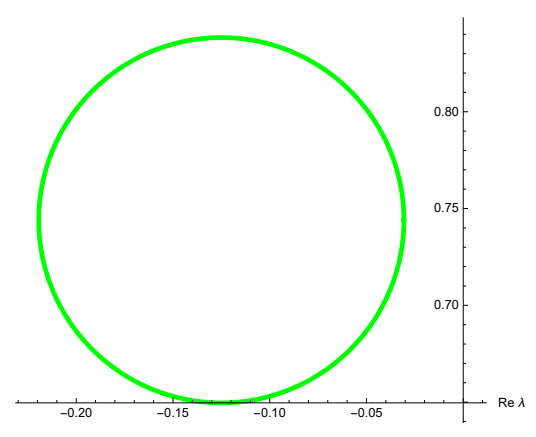

(a) $\mathcal{T}$

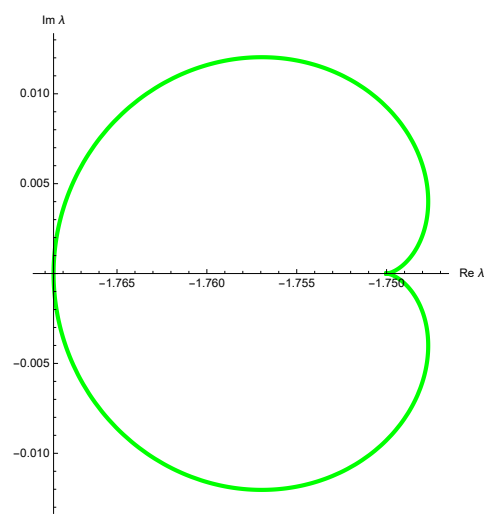

(b) $\mathcal{H}$

Figure 7. Curves $\mathcal{T}$ and $\mathcal{H}$ respectively resembling $\mathcal{B}_{1}$ and $\mathcal{B}_{3}$.

We now seek period-3 points on the boundary of $\boldsymbol{H}_{3}$ using $\lambda(t)$ in Equation (19) and $a$. Let $K_{4}$ denote the left side of Equation (17). Then we can write

$$
\left.K_{6}=\frac{1}{\lambda}\left[K_{4} \cdot\left(z^{2}+z+\lambda\right)+a \lambda+z\left(-1+a-\lambda^{2}\right)+z^{2}(-1+a-\lambda)-z^{3} \lambda\right)\right]
$$

to find Lemma 5 below:

Lemma 5. Period-3 point $z$ satisfies the following for a given $\lambda \neq 0$ and $a$.

$$
a \lambda+z\left(-1+a-\lambda^{2}\right)+z^{2}(-1+a-\lambda)-z^{3} \lambda=0 .
$$

By solving Equation (30) for $z$ in terms of $t \in[0,2 \pi$ ) exactly, we find three roots as the desired period-3 points:

$$
\left\{\begin{array}{l}
z^{(1)}(t)=\frac{1}{3}\left(\frac{\left.a-1-2^{-1 / 3} \beta_{1}-\frac{2^{-1 / 3} \beta_{2}}{\beta_{1}}-1\right),}{\lambda}-1\right) \\
z^{(2)}(t)=\frac{1}{3}\left(\frac{a-1+2^{-4 / 3}(1-i \sqrt{3}) \beta_{1}+\frac{2^{-2 / 3}(1+i \sqrt{3}) \beta_{2}}{\beta_{1}}}{\lambda}-1\right), \\
z^{(3)}(t)=\frac{1}{3}\left(\frac{a-1+2^{-4 / 3}(1+i \sqrt{3}) \beta_{1}+\frac{2^{-2 / 3}(1-i \sqrt{3}) \beta_{2}}{\beta_{1}}}{\lambda}-1\right),
\end{array}\right.
$$

where $a=\frac{1}{8} e^{i t}, \beta_{1}=\left(\omega_{1}+\sqrt{\omega_{2}}\right)^{1 / 3}, \beta_{2}=(a-1)^{2}+(a-1) \lambda+\lambda^{2}-3 \lambda^{3}, \omega_{1}=-2(a-$ $1)^{3}-3(a-1)^{2} \lambda+3(a-1) \lambda^{2}+(-7-18 a) \lambda^{3}-9 \lambda^{4}$ and $\omega_{2}=-4 \beta_{2}^{3}+\omega_{1}^{2}$.

Table 2 displays one of the typical period-3 points $z(t)$ at $t=\frac{2 \pi \ell}{k}$ highlighted in blue under the horizontal bar, together with the corresponding $\ell / k$-bifurcation point (marked by arrow lines in Figure 8) highlighted in red above the horizontal bar, for given specific values of $\ell$ and $k$. The types of $0 / 1-$ and $1 / 2$ - bifurcation points are fold and flip, respectively. Neimark-Sacker bifurcation occurs at all other $\ell / k$-bifurcation points. Besides, 
it is interesting to observe the $0 / 1$-root point with $k=1$ as a cusp point for the primitive component $\boldsymbol{H}_{3}^{(p)}$ illustrated in Figure 8e.

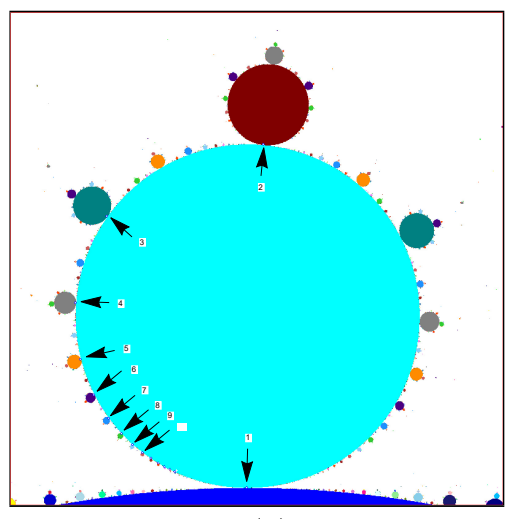

(a) $\lambda_{\ell, k} \in \partial \boldsymbol{H}_{3}^{\left(s_{1}\right)}, 1 \leq k \leq 10$

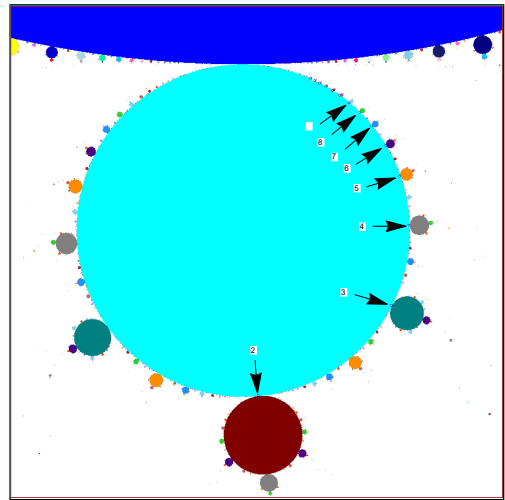

(c) $\lambda_{\ell, k} \in \partial \boldsymbol{H}_{3}^{\left(s_{2}\right)}, 1 \leq k \leq 10$

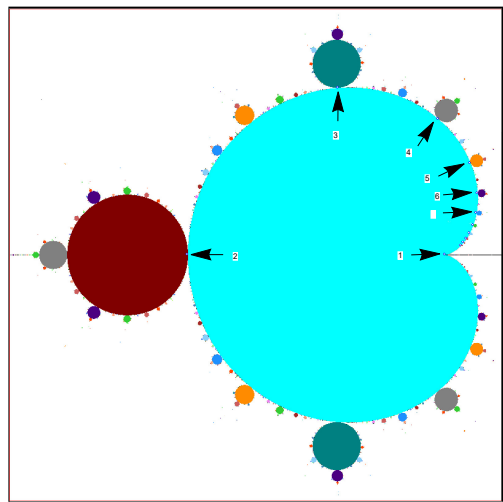

(e) $\lambda_{\ell, k} \in \partial \boldsymbol{H}_{3}^{(p)}, 1 \leq k \leq 7$

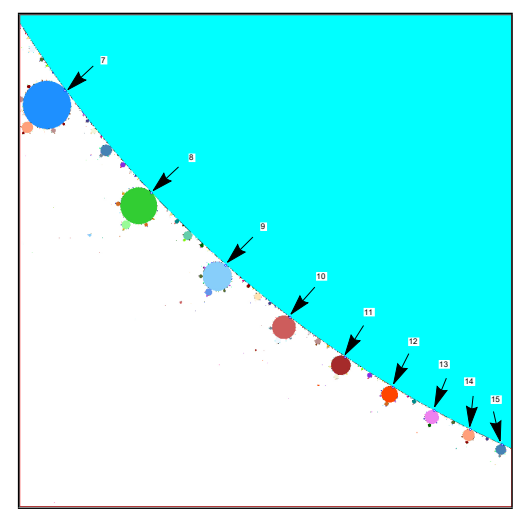

(b) $\lambda_{\ell, k} \in \partial \boldsymbol{H}_{3}^{\left(s_{1}\right)}, 7 \leq k \leq 15$

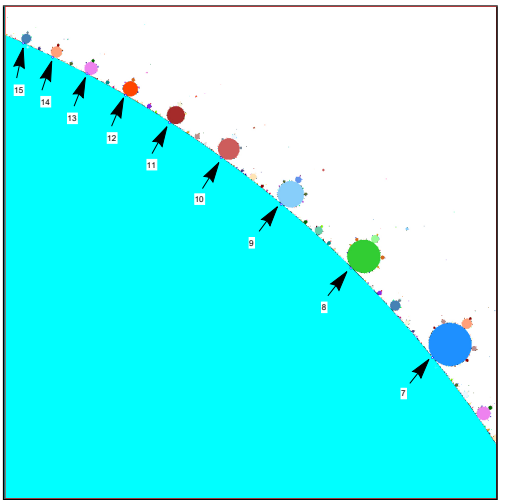

(d) $\lambda_{\ell, k} \in \partial \boldsymbol{H}_{3}^{\left(s_{2}\right)}, 7 \leq k \leq 15$

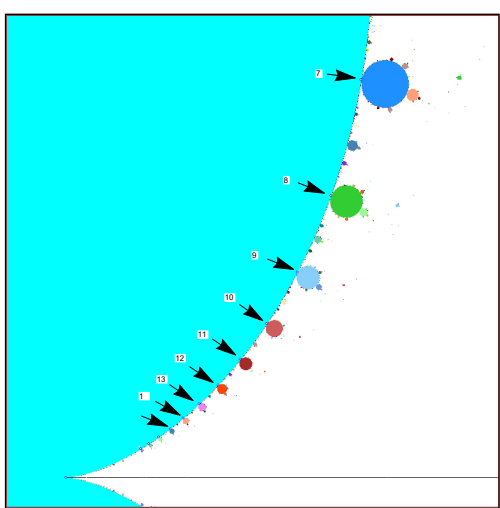

(f) $\lambda_{\ell, k} \in \partial \boldsymbol{H}_{3}^{(p)}, 7 \leq k \leq 15$

Figure 8. Bifurcation points $\lambda_{\ell, k}$ occurring on $\partial \boldsymbol{H}_{3}$. 
Table 2. Typical $\frac{\lambda_{\ell, k}}{\xi_{\ell, k}}$ along $\mathcal{B}_{1}$ and $\mathcal{B}_{3}$ for $1 \leq k \leq 15$ and $0 \leq \ell \leq 14$.

\begin{tabular}{|c|c|c|c|c|c|c|}
\hline$k$ & $\lambda_{b}^{(3)}$ & $\ell$ & $\lambda_{b}^{(1)}$ & $\ell$ & $\lambda_{a}^{(3)}$ & $\ell$ \\
\hline 1 & $\frac{\left(\begin{array}{c}-0.125 \\
0.649519\end{array}\right)}{\left(\begin{array}{c}-0.25 \\
0.433012\end{array}\right)}$ & 0 & $\frac{\left(\begin{array}{c}-0.125 \\
-0.649519\end{array}\right)}{\left(\begin{array}{c}-0.25 \\
-0.433012\end{array}\right)}$ & 0 & $\frac{-1.75}{1.301937}$ & 0 \\
\hline 2 & $\frac{\left(\begin{array}{c}-0.115735 \\
0.837999\end{array}\right)}{\left(\begin{array}{c}-0.768399 \\
0.630449\end{array}\right)}$ & 1 & $\frac{\left(\begin{array}{l}-0.115735 \\
-0.837999\end{array}\right)}{\left(\begin{array}{l}-0.768399 \\
-0.630449\end{array}\right)}$ & 1 & $\frac{-1.768529}{1.349439}$ & 1 \\
\hline 3 & $\frac{\left(\begin{array}{c}-0.201871 \\
0.798702\end{array}\right)}{\left(\begin{array}{c}0.160390 \\
-0.0267385\end{array}\right)}$ & 2 & $\frac{\left(\begin{array}{c}-0.0404292 \\
-0.786653\end{array}\right)}{\left(\begin{array}{c}-0.0607069 \\
-0.802461\end{array}\right)}$ & 2 & $\frac{\left(\begin{array}{c}-1.757699 \\
0.0120486\end{array}\right)}{\left(\begin{array}{c}1.336313 \\
-0.0215192\end{array}\right)}$ & 1 \\
\hline 4 & $\frac{\left(\begin{array}{c}-0.219192 \\
0.750878\end{array}\right)}{\left(\begin{array}{c}0.169206 \\
0.0497777)\end{array}\right)}$ & 3 & $\frac{\left(\begin{array}{c}-0.0303264 \\
-0.741102\end{array}\right)}{\left(\begin{array}{c}-0.0310562 \\
-0.771235\end{array}\right)}$ & 3 & $\frac{\left(\begin{array}{c}-1.750480 \\
0.00977578\end{array}\right)}{\left(\begin{array}{c}1.32375 \\
-0.0240248\end{array}\right)}$ & 1 \\
\hline 5 & $\frac{\left(\begin{array}{c}-0.216358 \\
0.719846\end{array}\right)}{\left(\begin{array}{l}0.159355 \\
0.101092\end{array}\right)}$ & 4 & $\frac{\left(\begin{array}{c}-0.0354688 \\
-0.713230\end{array}\right)}{\left(\begin{array}{c}-0.161133 \\
0.0931346\end{array}\right)}$ & 4 & $\frac{\left(\begin{array}{c}-1.748172 \\
0.00661584\end{array}\right)}{\left(\begin{array}{c}1.316444 \\
-0.0222350\end{array}\right)}$ & 1 \\
\hline 6 & $\frac{\left(\begin{array}{c}-0.208841 \\
0.700364\end{array}\right)}{\left(\begin{array}{l}0.145047 \\
0.136883\end{array}\right)}$ & 5 & $\frac{\left(\begin{array}{c}-0.0434897 \\
-0.695931\end{array}\right)}{\left(\begin{array}{c}-0.0130658 \\
-0.720166\end{array}\right)}$ & 5 & $\frac{\left(\begin{array}{c}-1.747668 \\
0.00443240\end{array}\right)}{\left(\begin{array}{c}1.312151 \\
-0.0198534\end{array}\right)}$ & 1 \\
\hline 7 & $\frac{\left(\begin{array}{c}-0.200936 \\
0.687714\end{array}\right)}{\left(\begin{array}{l}0.130401 \\
0.163072\end{array}\right)}$ & 6 & $\frac{\left(\begin{array}{c}-0.0513222 \\
-0.684671\end{array}\right)}{\left(\begin{array}{l}-0.542915 \\
-0.668132\end{array}\right)}$ & 6 & $\frac{\left(\begin{array}{c}-1.747740 \\
0.00304365\end{array}\right)}{\left(\begin{array}{c}1.309482 \\
-0.0176754\end{array}\right)}$ & 1 \\
\hline 8 & $\frac{\left(\begin{array}{c}-0.193766 \\
0.679151\end{array}\right)}{\left(\begin{array}{l}0.116641 \\
0.183049\end{array}\right)}$ & 7 & $\frac{\left(\begin{array}{c}-0.0582593 \\
-0.676996\end{array}\right)}{\left(\begin{array}{c}-0.214803 \\
0.0198685\end{array}\right)}$ & 7 & $\frac{\left(\begin{array}{c}-1.747973 \\
0.00215531\end{array}\right)}{\left(\begin{array}{c}1.307726 \\
-0.0158270\end{array}\right)}$ & 1 \\
\hline 9 & $\frac{\left(\begin{array}{c}-0.187534 \\
0.673126\end{array}\right)}{\left(\begin{array}{l}0.104066 \\
0.198816\end{array}\right)}$ & 8 & $\frac{\left(\begin{array}{c}-0.0642364 \\
-0.671554\end{array}\right)}{\left(\begin{array}{c}-0.223474 \\
0.00169750\end{array}\right)}$ & 8 & $\frac{\left(\begin{array}{c}-1.748228 \\
0.00157189\end{array}\right)}{\left(\begin{array}{c}1.306515 \\
-0.0142822\end{array}\right)}$ & 1 \\
\hline 10 & $\frac{\left(\begin{array}{c}-0.182180 \\
0.668744\end{array}\right)}{\left(\begin{array}{c}0.0926723 \\
0.211610\end{array}\right)}$ & 9 & $\frac{\left(\begin{array}{c}-0.0693574 \\
-0.667567)\end{array}\right)}{\left(\begin{array}{c}-0.230024 \\
-0.0142098\end{array}\right)}$ & 9 & $\frac{\left(\begin{array}{c}-1.748462 \\
0.00117702\end{array}\right)}{\left(\begin{array}{c}1.305646 \\
-0.0129885\end{array}\right)}$ & 1 \\
\hline 11 & $\frac{\left(\begin{array}{c}-0.177579 \\
0.665465\end{array}\right)}{\left(\begin{array}{c}0.0823571 \\
0.222229\end{array}\right)}$ & 10 & $\frac{\left(\begin{array}{l}-0.0737559 \\
-0.664563\end{array}\right)}{\left(\begin{array}{l}-0.497543 \\
-0.639443\end{array}\right)}$ & 10 & $\frac{\left(\begin{array}{c}-1.748664 \\
0.000901937\end{array}\right)}{\left(\begin{array}{c}1.305002 \\
-0.0118965\end{array}\right)}$ & 1 \\
\hline 12 & $\frac{\left(\begin{array}{c}-0.173608 \\
0.662951\end{array}\right)}{\left(\begin{array}{c}0.0729969 \\
0.231210\end{array}\right)}$ & 11 & $\frac{\left(\begin{array}{c}-0.0775554 \\
-0.662246\end{array}\right)}{\left(\begin{array}{l}-0.490190 \\
-0.633902\end{array}\right)}$ & 11 & $\frac{\left(\begin{array}{c}-1.748835 \\
0.000705185\end{array}\right)}{\left(\begin{array}{c}1.304512 \\
-0.0109662\end{array}\right)}$ & 1 \\
\hline 13 & $\frac{\left(\begin{array}{c}-0.170160 \\
0.660984\end{array}\right)}{\left(\begin{array}{c}0.0644724 \\
0.238925\end{array}\right)}$ & 12 & $\frac{\left(\begin{array}{c}-0.0808597 \\
-0.660423\end{array}\right)}{\left(\begin{array}{c}-0.242320 \\
-0.0520210\end{array}\right)}$ & 12 & $\frac{\left(\begin{array}{c}-1.748979 \\
0.000561120\end{array}\right)}{\left(\begin{array}{c}1.304131 \\
-0.0101659\end{array}\right)}$ & 1 \\
\hline 14 & $\frac{\left(\begin{array}{c}-0.167146 \\
0.659416\end{array}\right)}{\left(\begin{array}{c}-0.224273 \\
0.687261\end{array}\right)}$ & 13 & $\frac{\left(\begin{array}{c}-0.0837533 \\
-0.658963\end{array}\right)}{\left(\begin{array}{c}-0.244946 \\
-0.0621836\end{array}\right)}$ & 13 & $\frac{\left(\begin{array}{c}-1.749100 \\
0.000453409\end{array}\right)}{\left(\begin{array}{c}1.303828 \\
-0.00947141\end{array}\right)}$ & 1 \\
\hline 15 & $\frac{\left(\begin{array}{c}-0.164492 \\
0.658148\end{array}\right)}{\left(\begin{array}{c}0.0495185 \\
0.251555\end{array}\right)}$ & 14 & $\frac{\left(\begin{array}{c}-0.0863044 \\
-0.657776\end{array}\right)}{\left(\begin{array}{c}-0.247113 \\
-0.0714297\end{array}\right)}$ & 14 & $\frac{\left(\begin{array}{c}-1.749202 \\
0.000371375\end{array}\right)}{\left(\begin{array}{c}1.303584 \\
-0.00886359\end{array}\right)}$ & 1 \\
\hline
\end{tabular}

The expression $\frac{\lambda_{\ell, k}}{\xi_{\ell, k}}$ implies a pair of values $\ell / k$-root point $\lambda_{\ell, k}$ and period-3k point $\xi_{\ell, k}$.

\section{Conclusions}

When two components osculate each other, the governing Equation (9) of the osculating point obtained by an analysis from the Euclidean geometric point of view has played an innovative role in analyzing the geometry of the periodic components in Mandelbrot set $\mathscr{M}$. The theoretical boundary equation of the period-3 component is expected to help us understand the more detailed geometrical structure of $\mathscr{M}$. Without the knowledge of the theoretical boundary equation, it would have not been possible to exactly obtain the budding point and period- $3 k$ point of the period- $3 k$ component arising from the period-3 component.

As a future work, the current approach will be attempted to extend for some geometrical properties of period- $q$ mother-ship components with $q \geq 4$ and for a higher value of $k$, 
in which case one would encounter difficulties in establishing the boundary equation due to the higher complexity of composition task of $\Phi_{\lambda}^{q k}(z)$.

Author Contributions: Formal analysis, Y.-I.K.; investigation, Y.-H.G. Both authors have read and agreed to the final version of the manuscript.

Funding: The first author (Y.-H.G.) was partially supported by research grant NRF-2021R1A2C1012922 funded by the Korea government (MSIT).

Institutional Review Board Statement: Not applicable.

Informed Consent Statement: Not applicable.

Data Availability Statement: Not applicable.

Acknowledgments: We authors would like to express our sincere gratitude to the anonymous reviewers for providing valuable corrections and suggestions as well as correct vocabularies and English grammar.

Conflicts of Interest: The authors declare no conflict of interest.

\section{References}

1. Gulick, D. Encounters with Chaos; McGraw-Hill Inc.: New York, NY, USA, 1992.

2. Holden, A.V. Chaos; Princeton University Press: Princeton, NJ, USA, 1986.

3. Peitgen, H.; Richter, P. The Beauty of Fractals. Images of Complex Dynamical Systems; Springer: Berlin, Germany, 1986.

4. Campos, B.; Cordero, A.; Torregrosa, J.R.; Vindel, P. Orbits of period two in the family of a multipoint variant of Chebyshev-Halley family. Numer. Algorithms 2016, 73, 141-156. [CrossRef]

5. Behl, R.; Cordero, A.; Motsa, S.; Torregrosa, J.; Kanwar, V. An optimal fourth-order family of methods for multiple roots and its dynamics. Numer. Algorithms 2016, 71, 775-796. [CrossRef]

6. Geum, Y.H.; Kim, Y.I. A two-parameter family of fourth-order iterative methods with optimal convergence for multiple zeros. J. Appl. Math. 2013, 2013, 369067.

7. Magreñán, Á.A. Different anomalies in a Jarratt family of iterative root-finding methods. Appl. Math. Comput. 2014, 233, 29-38.

8. Neta, B.; Scott, M.; Chun, C. Basin attractors for various methods for multiple roots. Appl. Math. Comput. 2012, $218,5043-5066$. [CrossRef]

9. Wang, X.; Zhang, T.; Qin, Y. Efficient two-step derivative-free iterative methods with memory and their dynamics. Int. J. Comput. Math. 2016, 93, 1423-1446. [CrossRef]

10. Giarrusso, D.; Fisher, Y. A Parameterization of the Period 3 Hyperbolic Components of the Mandelbrot Set. Proc. Am. Math. Soc. 1995, 123, 3731-3737.

11. Carleson, L.; Gamelin, T.W. Complex Dynamics. In Universitext: Tracts in Mathematics; Springer: New York, NY, USA, 1993.

12. Ahlfors, L.V. Complex Analysis. An Introduction to the Theory of Analytic Functions of One Complex Variable; International Series in Pure and Applied Mathematics; McGraw-Hill Book Co.: New York, NY, USA, 1978.

13. Geum, Y.H.; Kim, Y.I. Long-Term Orbit Dynamics Viewed through the Yellow Main Component in the Parameter Space of a Family of Optimal Fourth-Order Multiple-Root Finders. Discret. Contin. Dyn. Syst.-B 2020, 25, 3087-3109.

14. Douady, A. Chaotic Dynamics and Fractals; Academic Press: Cambridge, MA, USA, 1986; 155p.

15. Brillinger, D.R. The Analyticity of the Roots of a Polynomial as Functions of the Coefficients. Math. Mag. 1966, $39,145-147$. [CrossRef]

16. Nayfeh, A.H.; Balachandran, B. Applied Nonlinear Dynamics: Analytical, Computational, and Experimental Methods; John Wiley \& Sons: New York, NY, USA, 2008.

17. Nickalls, R.W.D. Viete, Descartes, and the cubic equation. Math. Gaz. 2006, 90, 203-208. [CrossRef]

18. Uspensky, J.V. Theory of Equations; McGraw-Hill Book Co.: New York, NY, USA, 1948.

19. Wolfram, S. The Mathematica Book; Wolfram Media, Inc.: Champaign, IL, USA, 2003. 